\title{
Spherical polyelectrolyte brushes as carrier particles for proteins: An investigation of the structure of adsorbed and desorbed bovine serum albumin
}

\author{
G. Jackler ${ }^{\mathrm{a}}$, A. Wittemann ${ }^{\mathrm{b}}$, M. Ballauff ${ }^{\mathrm{b}}$ and C. Czeslik ${ }^{\mathrm{a}, *}$

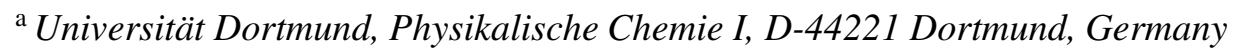 \\ ${ }^{\mathrm{b}}$ Universität Karlsruhe, Polymer-Institut, D-76128 Karlsruhe, Germany
}

\begin{abstract}
The change in the secondary and tertiary structure of bovine serum albumin (BSA) induced by the interaction with spherical polyelectrolyte brushes (SPB) has been investigated using fluorescence and circular dichroism (CD) spectroscopy. The SPB consist of poly(acrylic acid) chains grafted to a poly(styrene) core. The colloidal SPB represent a new substrate for protein immobilization because their protein binding capacity can be controlled by the ionic strength of the solution: SPB bind large amounts of BSA at low ionic strength $(\mathrm{pH}=6.1)$, but they are largely protein resistant at moderate salt concentrations of $500 \mathrm{mM}$. The conformation of BSA which was labeled with the environmentally sensitive dansyl fluorophore was studied before adsorption to the SPB, in the adsorbed state, and after desorption from the SPB. In the adsorbed state the obtained fluorescence spectrum is red-shifted which indicates a hydration of the dansyl fluorophores due to a distortion of the tertiary structure of BSA. Fluorescence and CD spectroscopic analysis of BSA that was desorbed from the SPB shows that the adsorption-induced conformational changes are largely reversible. Convex constraint analysis of the observed CD spectra of BSA yield $\alpha$-helix fractions of $68 \%$ and $57 \%$ before adsorption to and after desorption from the SPB, respectively. In a general view, the results of this study demonstrate that spherical polyelectrolyte brushes are suitable for a controlled immobilization and release of proteins without major conformational changes.
\end{abstract}

\section{Introduction}

Aqueous suspensions of colloidal particles represent excellent model systems for the study of the adsorption of dissolved proteins onto solid surfaces [1-7]. The colloidal size of the particles leads to an enormous surface area in the system that can be studied by a widespread variety of methods $[1,8]$. Moreover, the surface properties of latexes can be designed for special purposes by grafting a polymeric shell onto a nearly monodisperse core particle [8]. The surface of these particles obtained in this way is well-defined and can be changed for the purpose at hand. Results obtained from investigations of protein adsorption on such well-defined latex particles may therefore be compared to thorough studies conducted on macroscopic planar surfaces [9-18].

In a recent study, spherical polyelectrolyte brushes (SPB) have been presented as new and interesting colloid particles for the immobilization of proteins in solution [19]. The SPB consist of a solid core of

\footnotetext{
${ }^{*}$ Corresponding author: Dr. Claus Czeslik, University of Dortmund, Physical Chemistry I, Otto-Hahn-Str. 6, D-44227 Dortmund, Germany. Tel.: +49 231755 3903; Fax: +49 231755 3901; E-mail: claus.czeslik@uni-dortmund.de.
} 


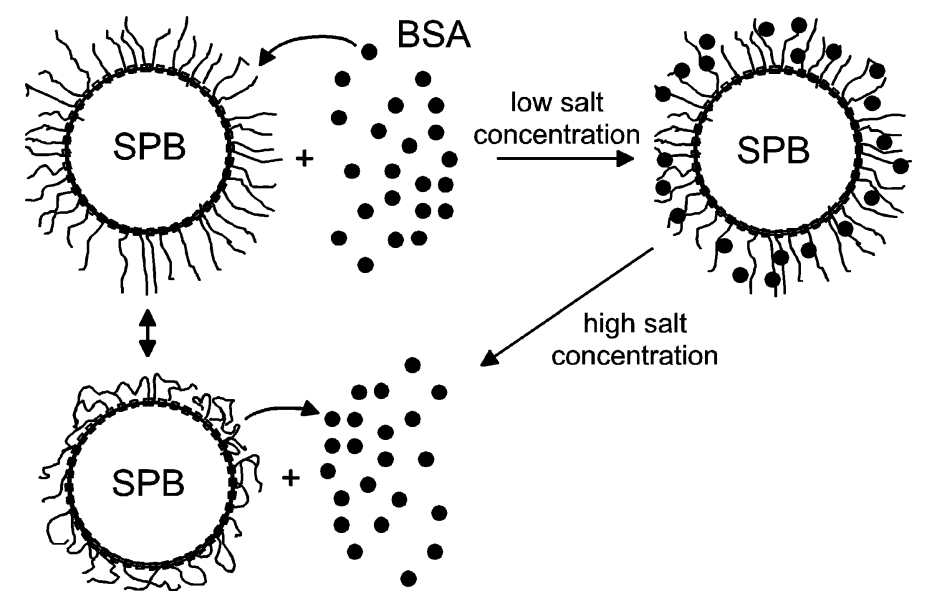

Fig. 1. Protein binding by spherical polyelectrolyte brushes. Strong adsorption of the dissolved proteins takes place at low ionic strength. Under these conditions the proteins are adsorbed irreversibly and no desorption takes place. However, if the salt concentration is raised to $0.5 \mathrm{M}$, the proteins are desorbed again and can be separated from the brush particles [19].

poly(styrene) onto which long linear polyelectrolyte chains of poly(acrylic acid) (PAA) or poly(styrene sulfonic acid) (PSS) are grafted [20-22]. The overall dimensions are in the colloidal domain (ca. 200$300 \mathrm{~nm}$ ) and have been characterized in detail recently [21,22]. Because of their colloidal size these particles generate a large surface area in solution that is well defined and highly suited for a systematic investigation of protein adsorption onto model surfaces. The SPB having long PAA chains on their surface exhibit a high binding capacity for protein molecules at low ionic strength even for proteins with a net negative charge [19]. In principle, the negative charge of the SPB should repel the negatively charged proteins at low ionic strength where the electrostatic repulsion is strongest. In contrast to this, strong adsorption takes place at low ionic strength and protein resistance is only observed at moderate salt concentrations. Moreover, bovine serum albumin (BSA) adsorbed at low ionic strength can almost quantitatively be removed from SPB with PAA chains by washing with a $500 \mathrm{mM}$ sodium chloride solution [19]. Thus, the ionic strength of the solution can be used as a physico-chemical switch for the protein affinity of the SPB. Figure 1 displays a scheme for the protein loading of the SPB taking place at low ionic strength.

The strong interaction of the spherical polyelectrolyte brushes with proteins in aqueous solution seems to be closely related to the binding of proteins by polyelectrolytes [23-28]. Here it has been found that protein adsorption takes place often on the "wrong side" of the isoelectric point where $\mathrm{pH}>\mathrm{pI}$ [27]. Dubin and coworkers have discussed this unexpected finding in terms of the dipolar asymmetry of the proteins [27]. Carlsson et al., however, have pointed to possible contributions of short-range attractive interactions that may lead to adsorption of proteins for $\mathrm{pH}>\mathrm{pI}$ [28]. In general, electrostatic attraction can be brought about by interaction of linear polyelectrolytes with heterogeneously charged surfaces [29]. The explanation for the strong adsorption of proteins onto the spherical polyelectrolyte brushes can therefore be based on the electrostatic interaction of the grafted polyelectrolyte chains and the positively charged patches of the protein [19]. These positively charged patches of the proteins become counterions of the polyelectrolyte brush and a concomitant number of ions is released. This leads to an increase of the entropy of the system and hence a marked confinement of the proteins within the grafted polyelectrolyte layer of the SPB ("counterion release force", see the discussion of this point in [30]) [19]. 
In this paper, we present the first study of conformational changes of a protein that is interacting with SPB in order to show the suitability of SPB for a controlled immobilization and release of proteins. Taking bovine serum albumin (BSA) as a model protein, detailed studies of the secondary and tertiary structure of the protein molecules before adsorption, in the adsorbed state and after a quantitative adsorption-desorption process are presented. BSA has a low isoelectric point of about $\mathrm{pH}=5[31,32]$, which has the advantage that a colloidal dispersion of the SPB at neutral $\mathrm{pH}$-values is not destabilized on the addition of BSA molecules. Almost 70\% of the 582 amino acid residues of BSA form $\alpha$-helices [32]. There are no $\beta$-sheets, but 17 disulfide bonds plus a free Cys side chain, 100 acidic residues (59 Glu and 41 Asp), and 99 basic residues (59 Lys, 23 Arg, and 17 His). In this study, BSA was labeled with the environmentally sensitive dansyl fluorophore [33], and its conformation was probed using fluorescence and CD spectroscopy. Unfortunately, due to the strong absorption and scattering of UV light by the SPB, no CD spectroscopy could be performed for BSA in the adsorbed state [34]. Since an SPB/water interface provides a reduced local $\mathrm{pH}$-value and an enhanced local ionic strength [19], additional experiments have been performed for comparison below $\mathrm{pH}=4$, where the so-called F-form of BSA is stable, and at different concentrations of sodium chloride [32]. Spectra were recorded as a function of temperature to characterize the thermal stability of the BSA conformations under the various conditions.

\section{Experimental}

Bovine serum albumin (BSA) was purchased from Fluka (Taufkirchen, Germany) and used as supplied. The protein molecules were labeled with dansyl fluorophores (Fluka, Taufkirchen, Germany) following the procedure described by Wang and Bright [35]. From the UV-absorbance at $279 \mathrm{~nm}$, which is characteristic for BSA, and that at $335 \mathrm{~nm}$, which is determined by the dansyl group, an average number of 1.4 dansyl groups per BSA molecule could be calculated. The purity of dansyl-BSA was checked by gel electrophoresis (SDS-PAGE) and found to be $>99 \%$; however, a small side band was observed indicating dimers. The tendency of fat-free albumin to aggregate is well known [31]. Dansyl-labeled BSA was used throughout this study to show that the label does not have any effect on the structure of BSA. Indeed, the CD spectra and the temperature of unfolding of dansyl-BSA are in excellent agreement with corresponding data of unlabeled BSA (see Results and discussion). Protein solutions with concentrations in the range of $0.02-0.5 \mathrm{mg} \mathrm{ml}^{-1}(0.3-7.5 \mu \mathrm{M})$ were prepared using a $10 \mathrm{mM}$ MES buffer, which was adjusted to $\mathrm{pH}=6.1$.

The spherical polyelectrolyte brushes that serve as substrate for BSA in this study consist of a poly(styrene) (PS) core and a poly(acrylic acid) (PAA) shell. They were prepared by photoemulsion polymerization as described before [20]. The radius of the PS core was $51 \mathrm{~nm}$, the contour length of the PAA chains was $36 \mathrm{~nm}$, and the grafting density was $0.13 \mathrm{~nm}^{-2}$ [19]. For adsorption studies, a few microliters of a 2.9 weight- $\%$ solution of the SPB were added to a BSA solution to obtain the desired mass ratio of BSA to SPB which was ranging from $0.4: 1$ to $2.0: 1$. The BSA/SPB solutions were equilibrated for at least $1 \mathrm{~h}$ prior to each measurement.

BSA that was first adsorbed to and then desorbed from the SPB was obtained as follows: Equilibrated solutions of BSA and SPB were filled in a serum replacement cell. Under gentle stirring, the solution was washed eight times with a pure buffer solution by passing the solution through a cellulose nitrate membrane (Schleicher \& Schuell, Kassel, Germany). The membrane had a pore size of $50 \mathrm{~nm}$ to retain the SPB with adsorbed BSA and to remove the loosely and non-adsorbed protein molecules from the solution. Then the whole procedure was repeated but this time the buffer solution contained sodium 
chloride with a concentration of $0.5 \mathrm{moll}^{-1}$ which leads to the desorption of the BSA from the SPB. The amount of protein washed off by this procedure was determined from UV absorption. From SDSPAGE, it has been found that the adsorption-desorption process did not increase the fraction of BSA dimers.

Static fluorescence measurements were performed using the K2 instrument from ISS (Champaign, Illinois, USA) which was operated in photon counting mode. The dansyl groups bound to BSA were excited with monochromatic light of $340 \mathrm{~nm}$ wavelength generated by a xenon arc lamp. Fluorescence spectra were recorded over the wavelength range $370-650 \mathrm{~nm}$. All fluorescence emission scans were normalized to the incident light intensity and were corrected for the wavelength-dependent response of the detection system. Background spectra were recorded and subtracted from the observed fluorescence spectra of dansyl-BSA. Data were analyzed using the Grams software from Galactic. Circular dichroism (CD) spectra were obtained using the J-715 instrument from Jasco (Tokyo, Japan). They were recorded over the wavelength range $193-250 \mathrm{~nm}$. The spectra are averages over $25-50$ scans acquired with $0.5 \mathrm{~nm}$ resolution and a scan speed of $100 \mathrm{~nm} \mathrm{~min}^{-1}$. The samples were contained in a $1 \mathrm{~mm}$ quartz cuvette, which was heated by a circulating water flow. A background spectrum of a MES buffer solution was recorded and found to have zero ellipticity at $193-250 \mathrm{~nm}$. The secondary structure of BSA was estimated by the convex constraint analysis (CCA, program version 1.0) [36,37]. In this analysis, a set of CD spectra is deconvoluted into its common pure-component curves over the wavelength range 195$240 \mathrm{~nm}$. It is noted that the CCA does not depend on known protein structures obtained from X-ray crystallography. In this study, the measured CD curves were appended to a CD data set of 26 globular proteins as used in [37]. The CD spectra were deconvoluted into 4 components (Fig. 2), which were assigned to $\alpha$-helix, $\beta$-sheet, turn and random coil secondary structures according to Fig. 2b of [36]. The RMS deviations between the measured CD spectra and those calculated from the component curves were found to be very small with a mean value of only 2.3 (RMS values below 10 are generally acceptable). The deviations were determined according to $100 \cdot\left[\sum_{i}\left(\theta_{\mathrm{obs}, i}-\theta_{\mathrm{calc}, i}\right)^{2} / \sum_{i} \theta_{\mathrm{obs}, i}^{2}\right]^{1 / 2}$ where $i$ is counting the data points of a CD spectrum, $\theta_{\mathrm{obs}, i}$ is the observed molar ellipticity and $\theta_{\text {calc }, i}$ is the

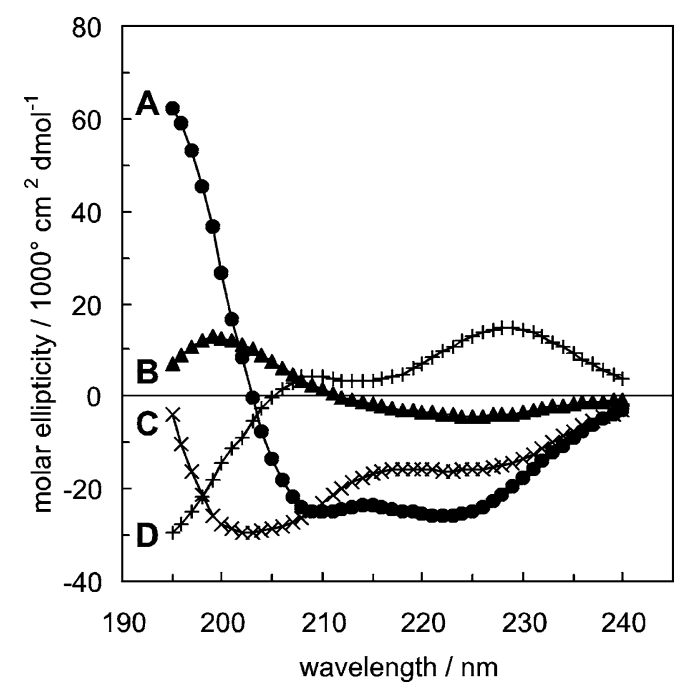

Fig. 2. Typical pure-component curves as obtained from the convex constraint analysis of the CD spectra. The curves are assigned to $\alpha$-helix (A), $\beta$-sheet (B), turn (C) and random coil (D) secondary structures following [36]. 
calculated molar ellipticity. All experiments have been performed at least twice. Experimental errors are given in the figure captions and the table heading.

\section{Results and discussion}

\subsection{Degree of adsorption}

In [19], the amount of BSA that is irreversibly adsorbed on the SPB has been determined for mass ratios of protein to polymer ranging from $0: 1$ to $1.4: 1$. At room temperature, essentially all BSA adsorbs up to a ratio of $0.5: 1$. In this study, we have determined the degree of BSA adsorption at a higher mass ratio in the excess of protein to investigate the effect of temperature on the degree of adsorption. A protein-polymer solution with the mass ratio $2: 1$ was centrifuged together with a protein solution $\left(0.2 \mathrm{mg} \mathrm{ml}^{-1}\right)$ as reference for $30 \mathrm{~min}$ at $55900 \mathrm{~g}$ and $20^{\circ} \mathrm{C}$. Then, the absorbance of the supernatant liquid of the protein-polymer sample was measured at $280 \mathrm{~nm}$ and compared to the corresponding signal of the reference. From this, a fraction of $33 \pm 5 \%$ could be calculated for BSA that is adsorbed on the spherical polyelectrolyte brushes. The degree of adsorption was also measured for protein-polymer samples that had been incubated at $60^{\circ} \mathrm{C}$ for $1 \mathrm{~h}$. A fraction of $47 \pm 3 \%$ of adsorbed protein was found from these measurements. These results demonstrate that there is no temperature-induced desorption of BSA from the SPB. Rather, an increase in temperature leads to an increased degree of adsorption which suggests that the adsorption of BSA on the SPB is dominated by entropic driving forces.

\subsection{Fluorescence spectroscopy}

In detailed fluorescence studies it has been shown that the dansyl fluorophor is an excellent probe for conformational changes of BSA in solution [35,38] and at polymeric interfaces [39]. The reason for this is the existence of two binding sites for the dansyl groups: the hydrophilic surface and the hydrophobic interior of BSA. Only the latter site contributes significantly to the observed fluorescence spectrum of dansyl-BSA. It can be identified by a blue-shifted spectrum with a maximum intensity at $450 \mathrm{~nm}$. In contrast, fully hydrated dansyl groups show a maximum fluorescence intensity at $550 \mathrm{~nm}$ [35]. Thus, a (partial) unfolding of dansyl-BSA leads to a red-shift of the dansyl-BSA fluorescence spectrum due to a hydration of the fluorophors.

In this study, the maximum of the fluorescence band of dansyl-BSA at $20^{\circ} \mathrm{C}$ has been found at $445 \mathrm{~nm}$ (Fig. 3) indicating that the dansyl groups have a hydrophobic environment and are located in the hydrophobic interior of BSA. With increasing temperature, the wavelength of maximum intensity is shifting to higher values reaching about $505 \mathrm{~nm}$ at $75^{\circ} \mathrm{C}$ (Fig. 3). This $60 \mathrm{~nm}$ red-shift of the emission maximum can be correlated with an increase of the average polarity surrounding the dansyl groups, which is induced by the thermal unfolding of the protein structure. When dansyl-BSA is adsorbed to the SPB, the same red-shift is observed and the fluorescence spectrum almost overlaps with that of thermally unfolded non-adsorbed dansyl-BSA (Fig. 3). A mass ratio of $0.4: 1$ for dansyl-BSA and SPB was used here, so that the fraction of non-adsorbed dansyl-BSA is negligible. This result indicates that the adsorption of dansyl-BSA on the spherical polyelectrolyte brushes leads to a distortion of the protein tertiary structure and a penetration of water into the protein interior.

The thermal unfolding of dansyl-BSA, with and without SPB, can be studied by plotting the fluorescence intensities observed at $435 \mathrm{~nm}$ as a function of temperature (Fig. 4). The wavelength of $435 \mathrm{~nm}$ has been chosen, because the fluorescence intensity is decreasing strongly at this wavelength during 


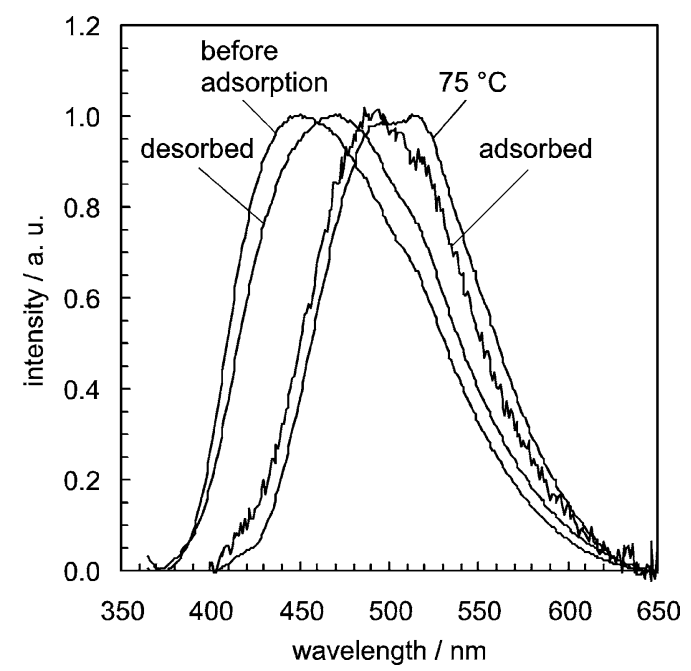

Fig. 3. Fluorescence spectra of dansyl-BSA solutions before adsorption, adsorbed to and desorbed from SPB at $20^{\circ} \mathrm{C}$ and $\mathrm{pH}=6.1$. A fluorescence spectrum of thermally unfolded dansyl-BSA at $75^{\circ} \mathrm{C}$ and $\mathrm{pH}=6.1$ is shown for comparison. The spectra are scaled to the same maximum intensity.

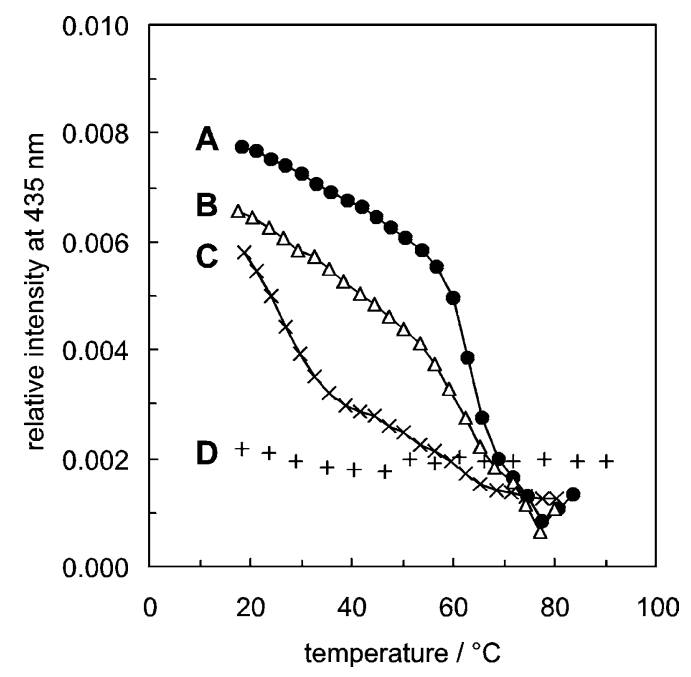

Fig. 4. Fluorescence intensity of dansyl-BSA at $435 \mathrm{~nm}$ as a function of temperature, normalized to the integral fluorescence intensity (A: before adsorption, B: after desorption from the SPB, C: at a reduced pH-value of 3.7, D: adsorbed to the SPB). Estimated errors for the intensities are $\pm 2 \cdot 10^{-4}$ for curves A, B, C and $\pm 5 \cdot 10^{-4}$ for curve D.

BSA unfolding (Fig. 3). Each intensity value shown has been normalized to the respective integral fluorescence intensity. The intensity of dansyl-BSA without SPB at $\mathrm{pH}=6.1$ (Fig. 4, curve A) shows a sigmoidal temperature dependence with a transition region at $60-63^{\circ} \mathrm{C}$ which locates the temperature of unfolding of the protein molecules. Using differential scanning calorimetry, the maximum differential heat capacity of a BSA solution at $\mathrm{pH}=7$ has been found at $62^{\circ} \mathrm{C}$ by Grasso and co-workers [40], in very good agreement with the temperature of unfolding observed in our study.

On the addition of SPB to a dansyl-BSA solution with a mass ratio of $0.4: 1$ (protein to polymer), where essentially all dansyl-BSA adsorbs on the SPB, no temperature dependence for the relative fluo- 


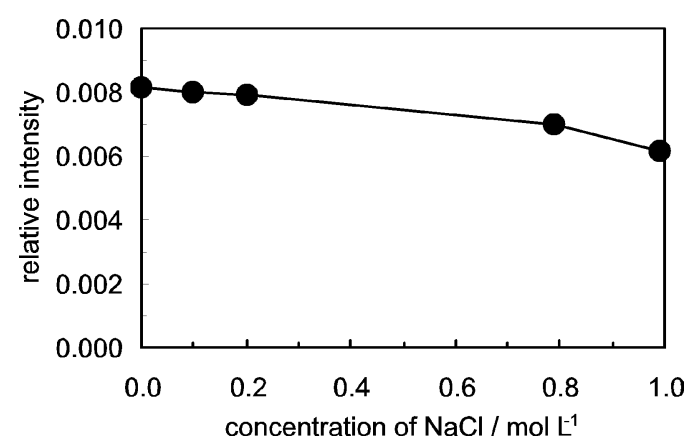

Fig. 5. Effect of sodium chloride on the relative fluorescence intensity at $435 \mathrm{~nm}$ of dansyl-BSA at $25^{\circ} \mathrm{C}$ and $\mathrm{pH}=6.1$. The intensities have an estimated error of $\pm 2 \cdot 10^{-4}$.

rescence intensity at $435 \mathrm{~nm}$ can be observed anymore at $18-90^{\circ} \mathrm{C}$ (Fig. 4, curve D). This behavior is a consequence of the distorted tertiary structure of adsorbed dansyl-BSA at low temperatures, which leads to a complete hydration of the dansyl fluorophores. Thus, the thermal unfolding of adsorbed dansyl-BSA cannot be probed anymore by the dansyl fluorescence.

The conformation of desorbed dansyl-BSA is characterized by the fluorescence spectrum given in Fig. 3 and curve B of Fig. 4. From these data it may be concluded that the conformational changes of dansyl-BSA that are induced by the interaction with the SPB are largely reversible. However, a small redshift of the fluorescence spectrum of desorbed dansyl-BSA relative to the spectrum of never-adsorbed dansyl-BSA remains which indicates an incomplete refolding of the protein molecules into their native tertiary structure (Fig. 3). The temperature dependence of the relative fluorescence intensity at $435 \mathrm{~nm}$ of desorbed dansyl-BSA (Fig. 4, curve B) shows the same temperature region for the thermal unfolding as that of never-adsorbed dansyl-BSA (Fig. 4, curve A), and both temperature-dependent curves overlap above about $65^{\circ} \mathrm{C}$. Thus, the thermal stability of dansyl-BSA is not altered significantly by a temporary interaction with the SPB.

Native BSA undergoes a transition to a modified conformation, the so-called F-form, as the $\mathrm{pH}$ value of the solution is lowered below 4.3 (there is a further transition to the so-called E-form at $\mathrm{pH}=2.7$ ) [31]. The F-form is probably characterized by an unfolded structure of one of the three protein domains. Although the SPB do not provide such an acidic environment for adsorbed dansylBSA molecules, the F-form of dansyl-BSA might be favored upon binding to the SPB. Therefore the fluorescence of dissolved dansyl-BSA in the F-form has also been analyzed at $\mathrm{pH}=3.7$ for comparison. The maximum fluorescence intensity of dansyl-BSA at $\mathrm{pH}=3.7$ is observed at about $479 \mathrm{~nm}$ (data not shown), clearly at shorter wavelength than the maximum fluorescence intensity of adsorbed dansyl-BSA at $491 \mathrm{~nm}$ (Fig. 3), which already indicates that adsorbed dansyl-BSA does not unfold into the F-form. This finding is supported by a comparison of the respective temperature dependences of the relative fluorescence intensities at $435 \mathrm{~nm}$ (Fig. 4, curves C and D). In contrast to adsorbed dansyl-BSA, dansyl-BSA in the F-form exhibits significant residual structure and is thermally unfolding in two steps showing an intermediate at about $40-65^{\circ} \mathrm{C}$, as can be inferred from the slope of curve $\mathrm{C}$ in Fig. 4.

Beside a reduced $\mathrm{pH}$-value there is an increased ionic strength within the shell of the SPB due to counterions [19]. Therefore the effect of an increased ionic strength on the fluorescence spectrum of dissolved dansyl-BSA has been investigated. In Fig. 5, the relative fluorescence intensity at $435 \mathrm{~nm}$ of dansyl-BSA is plotted for different concentrations of sodium chloride. Each intensity value is normalized to the respective integral fluorescence intensity. The data clearly show that there is no significant 

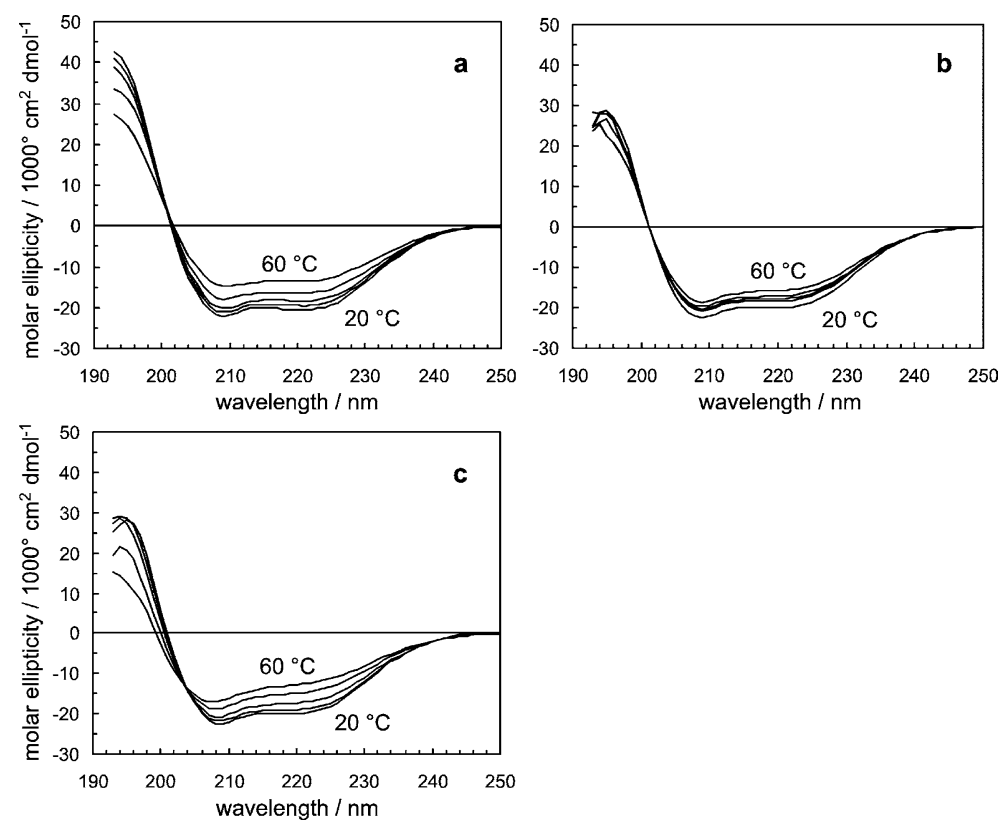

Fig. 6. CD spectra of BSA at $20-60^{\circ} \mathrm{C}$ before adsorption (a), desorbed from the SPB (b), and at a reduced pH-value of 3.4 (c). The CD spectra obtained at $20^{\circ} \mathrm{C}$ are typical for a protein structure with a large fraction of $\alpha$-helices (see Fig. 2 for comparison).

influence of sodium chloride on the fluorescence spectrum of dansyl-BSA at moderate salt concentrations. Indeed, the salt concentration within the SPB is estimated to be below 0.1 M [19]. Thus, it may be concluded that the observed adsorption-induced changes of the fluorescence of dansyl-BSA are not simply due to the different solvent properties as an increased ionic strength and a reduced $\mathrm{pH}$-value that are present within the SPB.

\subsection{Circular dichroism spectroscopy}

While the fluorescence experiments reported above mainly serve to detect changes in the tertiary structure of adsorbed BSA molecules, circular dichroism spectroscopy has been employed to analyze the effect of the spherical polyelectrolyte brushes on the secondary structure elements. In Fig. 6, CD spectra of BSA are shown for different temperatures before adsorption to the SPB, after desorption from the SPB, and at a reduced $\mathrm{pH}$-value of 3.4 (never adsorbed). By a comparison of the CD spectra of Fig. 6 with the pure-component curves displayed in Fig. 2, it is evident that the structure of BSA before adsorption and after desorption is dominated by $\alpha$-helices. However, there is a significant reduction of the molar ellipticity at $195 \mathrm{~nm}$ from about 40,000 before adsorption to about $30,000^{\circ} \mathrm{cm}^{2} \mathrm{dmol}^{-1}$ after desorption which indicates a small decrease of the $\alpha$-helix fraction after a complete adsorptiondesorption process.

In Table 1, the results of a secondary structure analysis of BSA are presented. As described in the Experimental section, the CD spectra were resolved by CCA into contributions of $\alpha$-helix, $\beta$-sheet, turn, and random coil structures. Fractions of secondary structure elements are given for BSA before adsorption, desorbed from the SPB, and at a reduced $\mathrm{pH}$-value of 3.4 (never adsorbed). At $20^{\circ} \mathrm{C}$ the recovered $\alpha$-helix fraction of dissolved BSA before adsorption is $68 \%$ (Table 1). This is in excellent agreement with other studies $[32,41]$. When increasing the temperature, this fraction is decreasing whereas the 
Table 1

Secondary structure of BSA before adsorption, desorbed from SPB, and at a reduced $\mathrm{pH}$-value

\begin{tabular}{|c|c|c|c|c|c|}
\hline \multirow[t]{2}{*}{$T\left({ }^{\circ} \mathrm{C}\right)$} & \multicolumn{4}{|c|}{ Fractions of secondary structure elements ${ }^{\mathrm{a}}(\%)$} & \multirow[t]{2}{*}{ RMS error ${ }^{b}$} \\
\hline & $\alpha$-helix & $\beta$-sheet & turn & random coil & \\
\hline \multicolumn{6}{|c|}{ before adsorption } \\
\hline 20 & 68 & 0 & 27 & 5 & 1.4 \\
\hline 30 & 66 & 3 & 26 & 5 & 1.2 \\
\hline 40 & 64 & 5 & 24 & 7 & 1.2 \\
\hline 50 & 59 & 9 & 22 & 10 & 1.8 \\
\hline \multicolumn{6}{|c|}{ desorbed from SPB } \\
\hline 20 & 57 & 1 & 36 & 5 & 4.2 \\
\hline 30 & 57 & 2 & 32 & 9 & 2.0 \\
\hline 40 & 56 & 3 & 31 & 10 & 2.2 \\
\hline 50 & 56 & 4 & 30 & 11 & 1.6 \\
\hline \multicolumn{6}{|c|}{ at $\mathrm{pH}=3.4$} \\
\hline 20 & 58 & 0 & 29 & 13 & 2.9 \\
\hline 30 & 56 & 0 & 29 & 15 & 1.6 \\
\hline 40 & 52 & 1 & 30 & 18 & 1.7 \\
\hline 50 & 43 & 4 & 32 & 20 & 2.3 \\
\hline
\end{tabular}

${ }^{a}$ Maximum errors for the fractions as derived from different experiments are $\pm 2 \%$ ( $\alpha$-helix), $\pm 2 \%$ ( $\beta$-sheet), $\pm 4 \%$ (turn), and $\pm 4 \%$ (random coil).

$\mathrm{b}$ Deviation between measured CD curve and CD curve calculated from the recovered purecomponent curves.

fractions of $\beta$-sheet and random coil structures are increasing. The observed higher $\beta$-sheet content at elevated temperatures indicates the formation of intermolecular $\beta$-sheets due to protein aggregation [42].

Desorbed BSA that was previously adsorbed to the SPB largely refolds into its native structure. Relative to native BSA, the $\alpha$-helix fraction of desorbed BSA is smaller by only $11 \%$, while the $\beta$-sheet fraction is similar and negligible (Table 1). It is noted that the observed loss of $\alpha$-helices is compensated by a formation of turns, while the random coil fraction remains unaltered (Table 1). With increasing temperature, the fraction of $\beta$-sheets of desorbed BSA is increasing which indicates protein aggregation, as found for BSA before adsorption (Table 1). However, there is no indication for a higher degree of aggregation after desorption which is a further indication for a native-like conformation of desorbed BSA. Apparently, in contrast to a solid substrate, the fluid-like SPB/water interface does not induce major conformational changes in proteins. Whereas on a solid surface only the protein can undergo conformational changes to optimize protein-surface interactions, contact optimization between a protein and SPB can be achieved by a rearrangement of the polyelectrolyte chains.

Finally, it is interesting to compare the partial unfolding of BSA when the $\mathrm{pH}$-value of the solution is lowered with the observed conformational changes that are induced by the interaction with SPB. As mentioned above, BSA forms the F-form between pH-values of 4.3 and 2.7 [31]. Although we do not expect such low $\mathrm{pH}$-values at the SPB/water interface, the F-form of BSA might still be favored due to interactions with the polyelectrolyte chains of the SPB. In Fig. $6 \mathrm{c}, \mathrm{CD}$ spectra of BSA at $\mathrm{pH}=3.4$ are shown and the secondary structures derived from these spectra are given in Table 1 . At $20^{\circ} \mathrm{C}$ and $\mathrm{pH}=$ 3.4, fractions of $58 \%$ for $\alpha$-helices, $0 \%$ for $\beta$-sheets, $29 \%$ for turns, and $13 \%$ for random coil structures have been found by CCA. These conformational weights are consistent with a partial unfolding of the protein by a decrease in the $\mathrm{pH}$-value, as seen in a shift from $\alpha$-helix to random coil structures. Since the 
interaction of BSA with the SPB leads to a partial transformation of $\alpha$-helices into turns, the results of the CD experiments clearly show that the conformation of BSA induced by the interaction with the SPB is not related to the F-form of BSA, in agreement with the fluorescence experiments reported above.

\section{Conclusions}

In this study we have presented the first conformational study of a protein that is interacting with spherical polyelectrolyte brushes (SPB). BSA was used as a model protein, which is characterized by a net negative charge at neutral $\mathrm{pH}$-values and a secondary structure consisting of $\alpha$-helices. The SPB have a poly(styrene) core and a poly(acrylic acid) shell and are characterized by a remarkable protein affinity at low ionic strength, which is drastically lowered as the ionic strength of the solution is raised. Using this tunable binding capacity of the SPB for protein molecules, the conformation of BSA was studied before adsorption, in the adsorbed state and after desorption. Adsorbed on the SPB, dansyl-BSA shows a red-shifted fluorescence spectrum which suggests a distorted protein tertiary structure allowing water to penetrate into the protein interior. Fluorescence and CD spectra of desorbed BSA that was previously adsorbed to the SPB reveal that the adsorption-induced conformational changes are largely reversible. The results of this study demonstrate that SPB are useful substrates for a controlled immobilization and release of proteins. Further studies of the adsorption of enzymes on the SPB, including the investigation of their biological activity, have now to be performed and are already under way.

\section{Acknowledgements}

We thank the Deutsche Forschungsgemeinschaft (DFG), the DFG-Center of Functional Nanostructures (CFN), and the Fonds der Chemischen Industrie for financial support.

\section{References}

[1] H. Kawaguchi, Functional polymer microspheres, Prog. Polym. Sci. 25 (2000), 1171-1210.

[2] W. Norde and J. Lyklema, The adsorption of human plasma albumin and bovine pancreas ribonuclease at negatively charged polystyrene surfaces, J. Colloid Interface Sci. 66 (1978), 257-302.

[3] C.A. Giacomelli, A.W.P. Vermeer and W. Norde, Adsorption of immunoglobulin G on core-shell latex particles precoated with chaps, J. Colloid Interface Sci. 231 (2000), 283-288.

[4] J.M. Peula, J. Callejas and F.J. de las Nieves, in: Surface Properties of Biomaterials, R. West and G. Batts, eds, Butterworth-Heinemann, Manchester, 1994.

[5] J.M. Peula and F.J. de las Nieves, Adsorption of monomeric bovine serum albumin on sulfonated polystyrene model colloids. 3. Colloidal stability of latex-protein complexes, Colloids Surf. A 90 (1994), 55-62.

[6] J.-Y. Yoon, J.-H. Kim and W.-S. Kim, The relationship of interaction forces in the protein adsorption onto polymeric microspheres, Colloids Surf. A 153 (1999), 413-419.

[7] J.-T. Oh and J.-H. Kim, Preparation and properties of immobilized amyloglucosidase on non-porous PS/PNaSS microspheres, Enzyme Microbial Technol. 27 (2000), 356-361.

[8] M. Ballauff, Nanoscopic polymer particles with a well-defined surface: synthesis, characterization, and properties, Macromol. Chem. Phys. 204 (2003), 220-234.

[9] M. Malmsten, ed., Biopolymers at Interfaces, Marcel Dekker, New York, 2003.

[10] T.A. Horbett and J.L. Brash, eds, Proteins at Interfaces II, American Chemical Society, Washington, DC, 1995.

[11] E. Ostuni, R.G. Chapman, R.E. Holmlin, S. Takayama and G.M. Whitesides, A survey of structure-property relationships of surfaces that resist the adsorption of protein, Langmuir 17 (2001), 5605-5620.

[12] E. Ostuni, B.A. Grzybowski, M. Mrksich, C.S. Roberts and G.M. Whitesides, Adsorption of proteins to hydrophobic sides on mixed self-assembled monolayers, Langmuir 19 (2003), 1861-1872, and further references given therein. 
[13] C.F. Wertz and M.M. Santore, Fibrinogen adsorption on hydrophilic and hydrophobic surfaces: geometrical and energetic aspects of interfacial relaxations, Langmuir 18 (2002), 706-715, and further references given therein.

[14] C.F. Wertz and M.M. Santore, Adsorption and reorientation kinetics of lysozyme on hydrophobic surfaces, Langmuir 18 (2002), 1190-1199.

[15] R.L.C. Wang, H.J. Kreuzer and M. Grunze, The interaction of oligo(ethylene oxide) with water: a quantum mechanical study, Phys. Chem. Chem. Phys. 2 (2000), 3613-3622, and further references given therein.

[16] S. Kidoaki and T. Matsuda, Mechanistic aspects of protein/material interactions probed by atomic force microscopy, Colloids Surf. B $\mathbf{2 3}$ (2002), 153-163.

[17] G. Jackler, R. Steitz and C. Czeslik, Effect of temperature on the adsorption of lysozyme at the silica/water interface studied by optical and neutron reflectometry, Langmuir 18 (2002), 6565-6570.

[18] C. Czeslik, C. Royer, T. Hazlett and W. Mantulin, Reorientational dynamics of enzymes adsorbed on quartz: a temperaturedependent time-resolved TIRF anisotropy study, Biophys. J. 84 (2003), 2533-2541.

[19] A. Wittemann, B. Haupt and M. Ballauff, Adsorption of proteins on spherical polyelectrolyte brushes in aqueous solution, Phys. Chem. Chem. Phys. 5 (2003), 1671-1677.

[20] X. Guo, A. Weiss and M. Ballauff, Synthesis of spherical polyelectrolyte brushes by photoemulsion polymerization, Macromolecules 32 (1999), 6043-6046.

[21] X. Guo and M. Ballauff, Spatial dimensions of colloidal polyelectrolyte brushes as determined by dynamic light scattering, Langmuir 16 (2000), 8719-8726.

[22] X. Guo and M. Ballauff, Spherical polyelectrolyte brushes: comparison between annealed and quenched brushes, Phys. Rev. E 64 (2001), 051406.

[23] W.A. Bowman, M. Rubinstein and J.S. Tan, Polyelectrolyte-gelatin complexation: light-scattering study, Macromolecules 30 (1997), 3262-3270.

[24] D. Takahashi, Y. Kubota, K. Kokai, T. Izumi, M. Hirata and E. Kokufuta, Effects of surface charge distribution of proteins in their complexation with polyelectrolytes in an aqueous salt-free system, Langmuir 16 (2000), 3133-3140.

[25] J. Xia, K. Mattison, V. Romano, P.L. Dubin, B.B. Muhoberac, Complexation of trypsin and alcohol dehydrogenase with poly(diallyldimethylammonium chloride), Biopolymers 41 (1997), 359-365.

[26] T. Hattori, R. Hallberg and P.L. Dubin, Roles of electrostatic interaction and polymer structure in the binding of $\beta$ lactoglobulin to anionic polyelectrolytes: measurement of binding constants by frontal analysis continuous capillary electrophoresis, Langmuir 16 (2000), 9738-9743.

[27] E. Seyrek, P.L. Dubin, C. Tribet and E.A. Gamble, Ionic strength dependence of protein-polyelectrolyte interactions, Biomacromolecules 4 (2003), 273-282.

[28] F. Carlsson, P. Linse and M. Malmsten, Monte Carlo simulations of polyelectrolyte-protein complexation, J. Phys. Chem. B 105 (2001), 9040-9049.

[29] M. Ellis, C.Y. Kong and M. Muthukumar, Polyelectrolyte adsorption on heterogeneously charged surfaces, J. Chem. Phys. 112 (2000), 8723-8729.

[30] C. Fleck and H.H. von Grünberg, Counterion evaporation, Phys. Rev. E. 63 (2001), 061804.

[31] T. Peters, Serum albumin, Adv. Protein Chem. 37 (1985), 161-245.

[32] D.C. Carter and J.X. Ho, Structure of serum albumin, Adv. Protein Chem. 45 (1994), 153-203.

[33] J.R. Lakowicz, Principles of Fluorescence Spectroscopy, Kluwer Academic/Plenum, New York, 1999.

[34] D.W. Urry, Protein conformation in biomembranes: optical rotation and absorption of membrane suspensions, Biochim. Biophys. Acta 265 (1972), 115-168.

[35] R. Wang and F.V. Bright, Rotational reorientation kinetics of dansylated bovine serum albumin, J. Phys. Chem. 97 (1993), 4231-4238.

[36] A. Perczel, M. Hollósi, G. Tusnády and G.D. Fasman, Convex constraint analysis: a natural deconvolution of circular dichroism curves of proteins, Protein Engin. 4 (1991), 669-679.

[37] A. Perczel, K. Park and G.D. Fasman, Analysis of the circular dichroism spectrum of proteins using the convex constraint algorithm: a practical guide, Anal. Biochem. 202 (1992), 83-93.

[38] R. Wang and F.V. Bright, Effects of protein denaturation on the rotational reorientation kinetics of dansylated bovine serum albumin, J. Phys. Chem. 97 (1993), 10872-10878.

[39] E.J. Bekos, J.P. Ranieri, P. Aebischer, J.A. Gardella and F.V. Bright, Structural changes of bovine serum albumin upon adsorption to modified fluoropolymer substrates used for neural cell attachment studies, Langmuir 11 (1995), 984-989.

[40] C. la Rosa, D. Milardi, S. Fasone and D. Grasso, A combined scanning dilatometric and differential scanning calorimetric study of the thermal unfolding of bovine serum albumin, Thermochim. Acta 235 (1994), 231-237.

[41] N. Kossovsky, A. Nguyen, K. Sukiassians, A. Festekjian, A. Gelman and E. Sponsler, Secondary structure of albumin acquired rapidly by modified conventional ATR-FTIR is comparable to CD spectral data, J. Colloid Interface Sci. 166 (1994), 350-355, and further references given therein.

[42] R.J. Green, I. Hopkinson and R.A.L. Jones, Unfolding and intermolecular association in globular proteins adsorbed at interfaces, Langmuir 15 (1999), 5102-5110. 


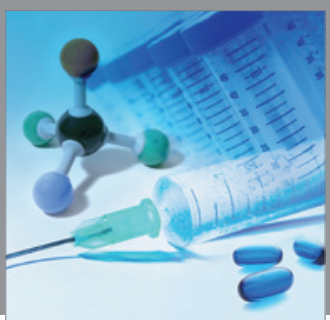

International Journal of

Medicinal Chemistry

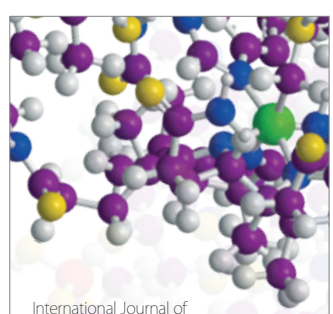

Carbohydrate Chemistry

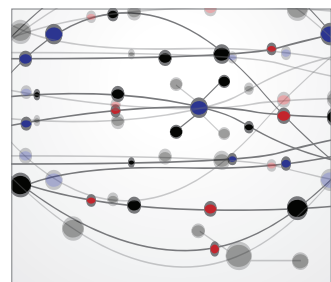

The Scientific World Journal
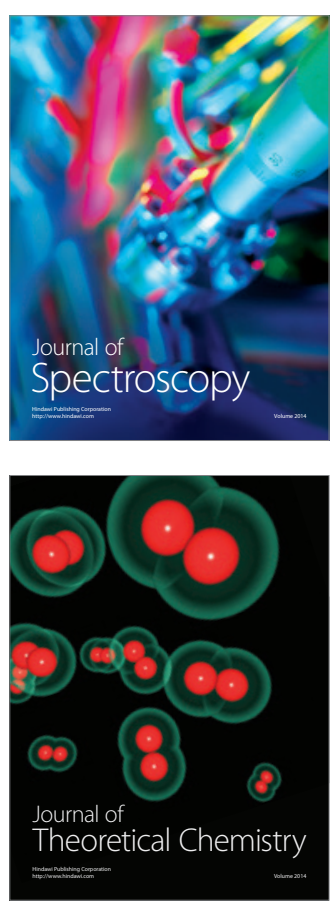
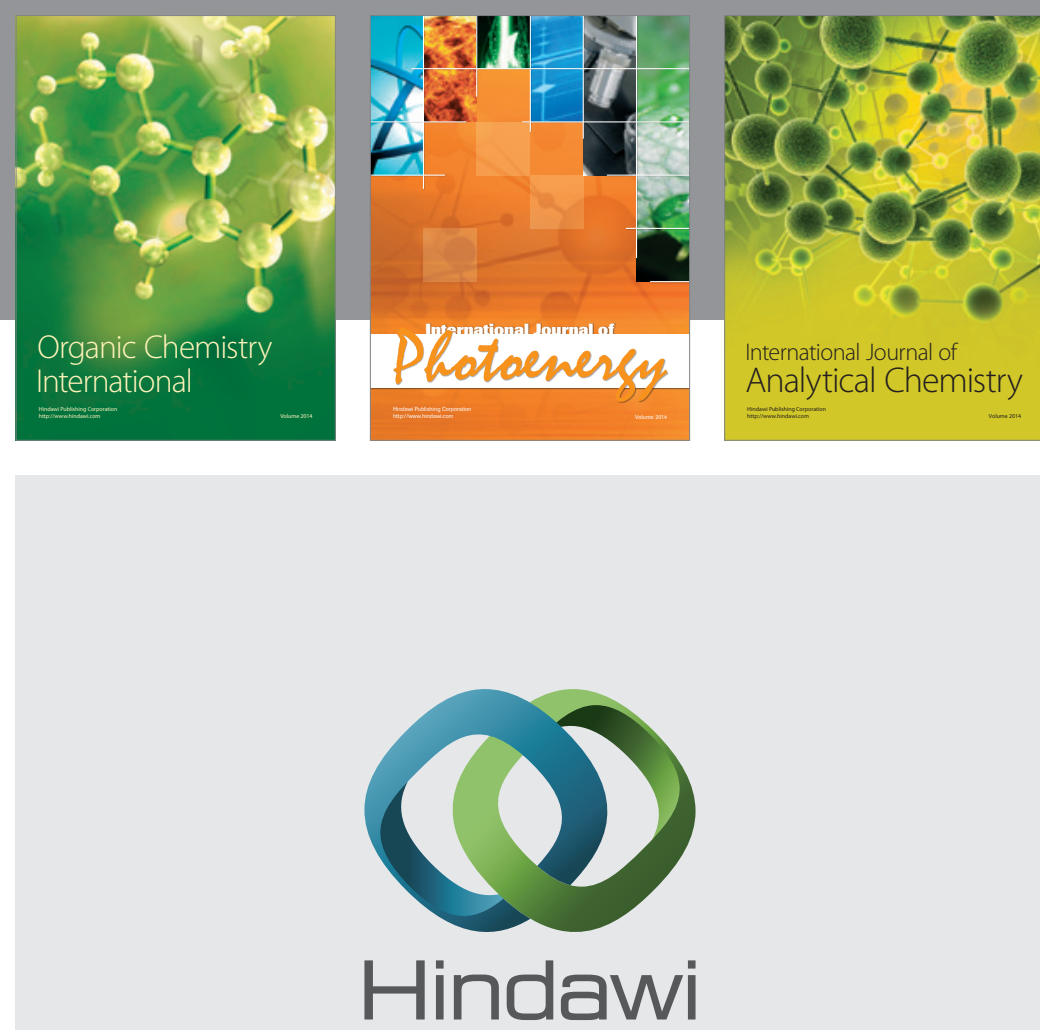

Submit your manuscripts at

http://www.hindawi.com
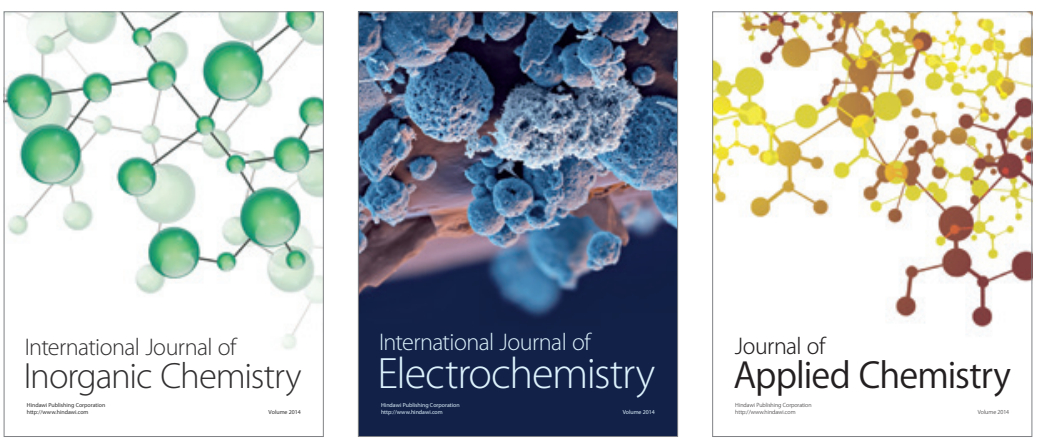

Journal of

Applied Chemistry
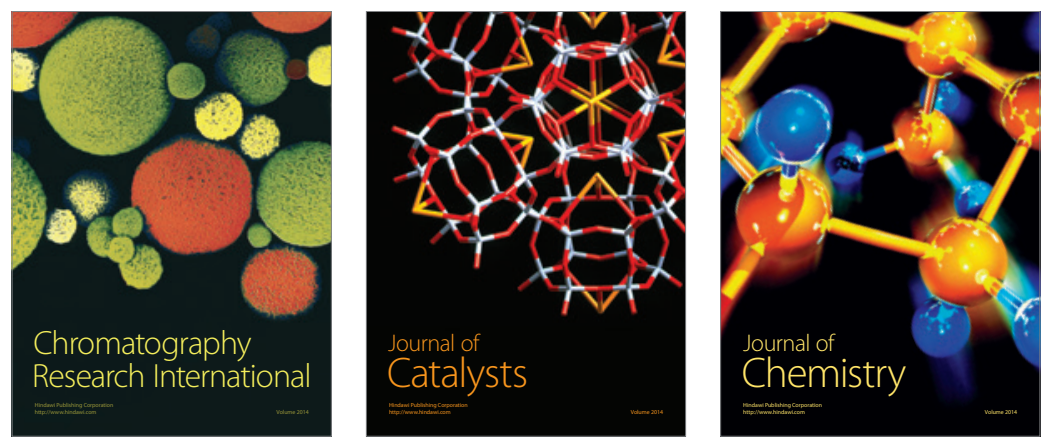
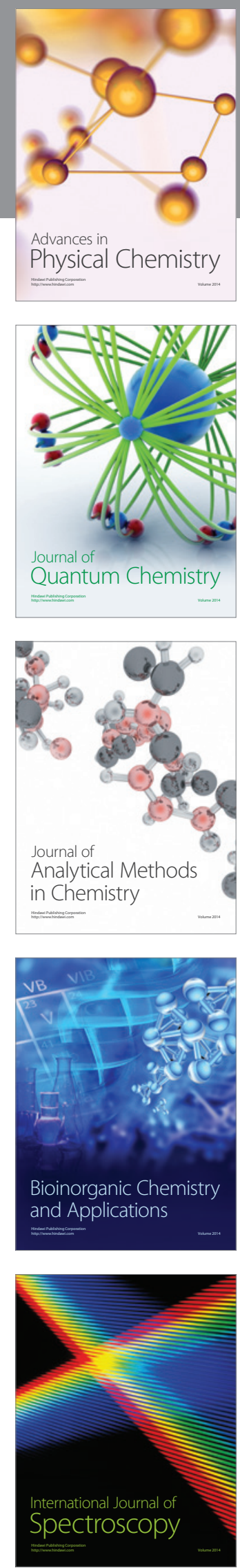